\title{
The interaction of bladder-filling behavior and ureteral function
}

\author{
Edward J. McGuire \\ Department of Surgery, Section of Urology, University of Michigan Medical Center, Ann Arbor, MI, USA
}

Summary. The interrelationship between bladder-filling behavior and ureteral delivery of urine into the bladder was studied in myelodysplastic children, in a primate neurogenic bladder-dysfunction model, and in a feline obstructive-uropathy model. All of these studies confirm the observation that intravesical pressure is the most important obstacle to ureteral delivery of urine into the bladder. Our concepts of ureteral function may need some revision. The findings in the obstructive feline model suggest that we should measure ureteral delivery of urine into the bladder in relation to intravesical pressure since this method may define ureteral reserve "power" better than either perfusion studies or dynamic renal scans.

The observation that ureteral function can be impaired by obstructive uropathy and various neurogenic conditions is not new, but exactly how this occurs is, surprisingly, not very well established. Clearly, not all patients with obstructive uropathy or neurogenic conditions develop ureteral dysfunction. However, radiography studies show that many patients with neurogenic conditions and some with obstructive uropathy do develop upper tract dysfunction if they are not treated. Treatment generally consists of efforts to improve bladder emptying by cutting the sphincter or part of the sphincter, by removing the prostate, or by using intermittent catheterization or constant drainage of the bladder by tubes, vesicostomy, or supravesical diversion. At least in the short term, these treatments can result in radiographic evidence of improvement in ureteral function, in that the ureters return to normal or become less dilated. Ureteral function in these conditions could be impaired because the obstructed or neurogenically impaired bladder develops "hypertrophy" and undergoes thickening and collagen deposition, which have a local compressive effect on the ureter combined with a kind of "tonic" effect related to activity in the bladder musculature. This process could interfere with the ability of the ureter to deliver urine into the bladder and with the valvular mechanism that prevents reflux at the ureteral terminus [2]. These changes could interfere with the antibacterial defense mechanism of the upper urinary tract, leading to problems with ureteral and renal function. Alternatively, the physical changes observed in the neurogenic or obstructive bladder that involve trabeculation and thickening of the bladder wall could be associated with increased intravesical pressure, which might result in ureteral dilation and vesicoureteral reflux, and could conceivably be the trigger mechanism for the observed morphological changes in the bladder itself [1].

\section{Experimental and clinical data}

Three separate studies were performed. The first was done over a 12 -year period in a large cohort of myelodysplastic children and involved some 350 individuals, the second investigated a small series of primates with complete sacral root ablation, and the third involved a large group of cats with obstructive uropathy due to an implanted periurethral silastic cuff.

Primates with complete sacral root and cord ablation were subjected to periodic urodynamic evaluation involving cystometrograms with pelvic-floor electromyograms (EMGs) over a period of 4 months, during which time the bladder had become thicker as determined by radiographic imaging and bladder compliance, defined as the volume required to cause leakage across the sphincter in the anesthetized (ketamine) animal, had diminished. At 4 months, the ureter was cannulated and perfused at a slow rate $(0.09 \mathrm{ml} / \mathrm{min})$ while the bladder was first constantly emptied and then progressively filled. The bladder outlet was occluded by a balloon and bladder pressures were measured constantly, as were ureteral pressures (Fig. 1). The ureter tolerated perfusion accompanied by continuous bladder emptying with no change in pressure. An increase in bladder pressure brought about by filling was immediately reflected in ureteral pressure, and this relationship was maintained over the entire range of in- 


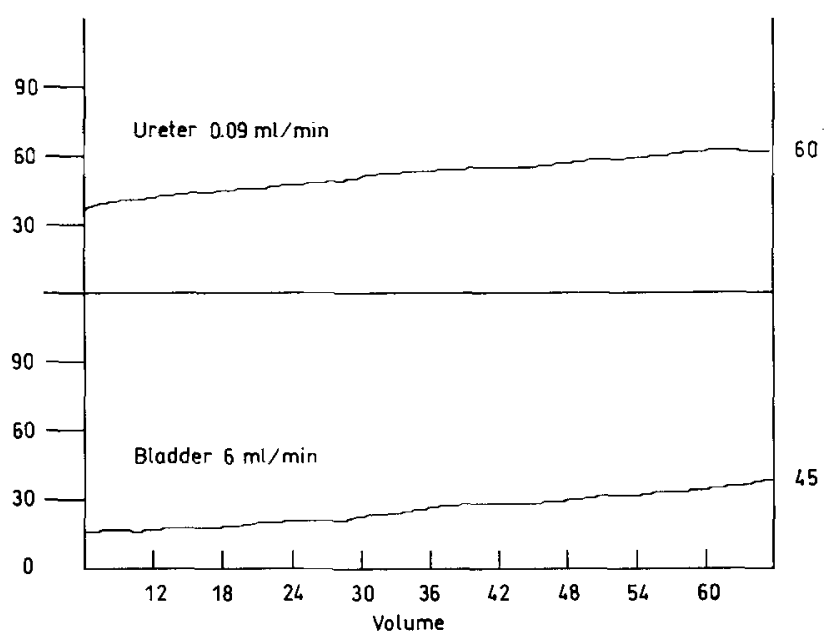

Fig. 1. Primate ureteral perfusion and bladder-pressure response (decentralized bladder). Note the parallel relationship between ureteral and bladder-pressure responses to perfusion

travesical pressures and volumes. In every instance, ureteral pressure paralleled bladder pressure precisely and was slightly higher than intravesical pressure. At the end of each experiment the animals were assessed for vesicoureteral reflux, which was found not to be present.

\section{Clinical studies in myelodysplasia}

A large group of children were followed urodynamically and radiographycally at the University of Michigan for 12 years. Beginning in 1983, all of these children were managed with the underlying goal of achieving a bladder

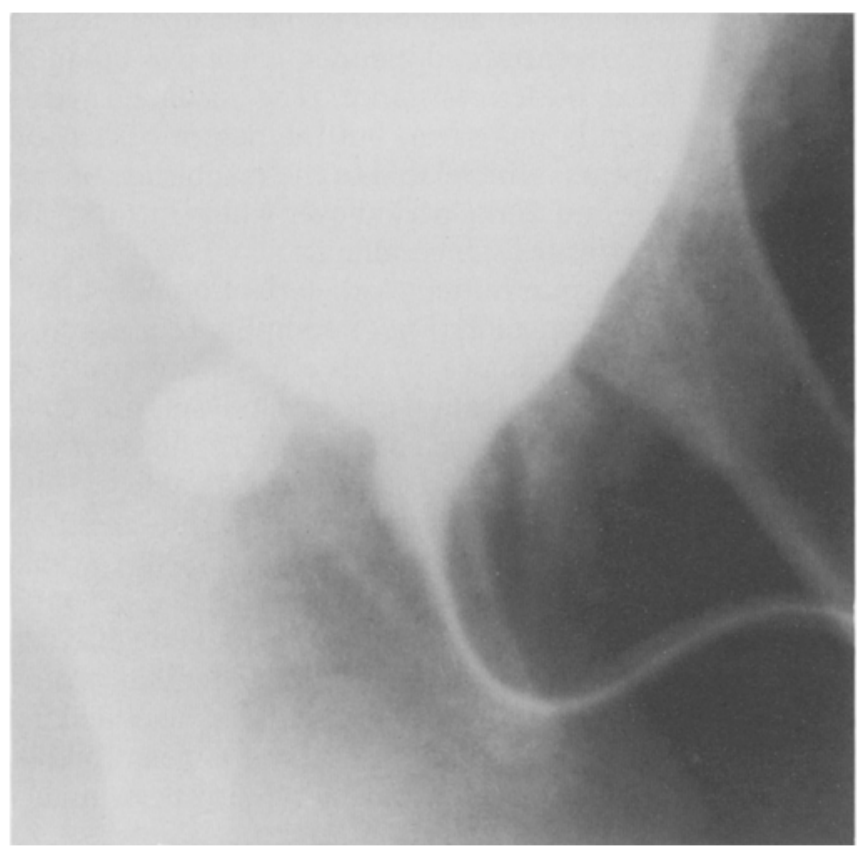

Fig. 2. Chronic (1-year) obstructed feline ureteral perfusion as compared with that of a normal cat. There is some increase in pressure required over the chronic obstruction pressure of $<40 \mathrm{~cm} \mathrm{H}_{2} \mathrm{O}$ in all circumstances. That is, at the time of leakage or at the volumes obtained by intermittent catheterization, measured bladder pressure had to be lower, preferably much lower, than $40 \mathrm{~cm} \mathrm{H}_{2} \mathrm{O}$. At some time during their course, about $40 \%$ of these children showed elevated bladder pressure during urinary storage or at the time of urethral urinary leakage. Patients who exhibited elevated bladder pressure showed either an areflexic bladder with a nonfunctional vesical outlet (Fig. 2) or a reflexive bladder with a discoordinate external sphincter but normal closure of the proximal sphincter. Of the entire group, a small number were "normal" with respect to lower urinary tract function $(5 \%) ; 85 \%$ showed an areflexic bladder and an open vesical outlet; and $10 \%$, a reflexive bladder with detrusor-sphincter dyssynergia.

The situation in patients with areflexic vesical dysfunction particularly lends itself to study since the relationship between bladder storage pressures and outlet resistance, which is virtually fixed, is linear. This simply means that the intrinsic intravesical pressure required to produce leakage is almost identical to the closing pressure of the urethra, despite the fact that most such patients have easily demonstrable stress incontinence and show minor changes in intra-abdominal pressure. Children with higher outlet resistance that has not been treated have a high incidence of development of ureteral dilation, vesicoureteral reflux, and renal parenchymal damage. No patient with upper tract disease demonstrated any bladder-filling pattern other than a very poorly compliant one. Thus, the initial findings suggested a relationship between outlet resistance and poor compliance as well as between outlet resistance and upper tract deterioration.

Since the primate data indicated that intravesical pressure was transmitted to the ureter irrespective of the presence or absence of reflux, it appeared that poor compliance in association with elevated outlet resistance resulted in a situation in which a sustained increase in intravesical pressure was possible and that this resulted in damage to the upper urinary tract. The clinical occurrence of this situation in a child with a dilated upper urinary tract prompts pediatric urologists to proceed with vesicostomy, which is almost invariably successful in improving ureteral appearance as judged by radiography. The functional result of vesicostomy is simply a reduction in bladder pressure rather than a more complicated outcome.

In our series, some 68 patients showed high outlet resistance, poor compliance, and sustained bladder pressure of $>40 \mathrm{~cm} \mathrm{H}_{2} \mathrm{O}$. Most (52) of these children responded to drugs and the institution of intermittent catheterization, exhibiting a satisfactory reduction in intravesical pressure and an improvement in upper tract function equivalent to that seen after vesicostomy. However, 13 children did not respond to intermittent catheterization and anticholinergic agents; in 2 of these cases a vesicostomy was performed, but the others underwent a simple urethral dilation to reduce the outlet resistance and lower the intravesical pressure. The outlet was dilated to cause leakage enough to reduce the intravesical pressure to $\leq 20 \mathrm{~cm} \mathrm{H}_{2} \mathrm{O}$. Follow-up cystometrograms demonstrated a striking improvement in compliance that was 


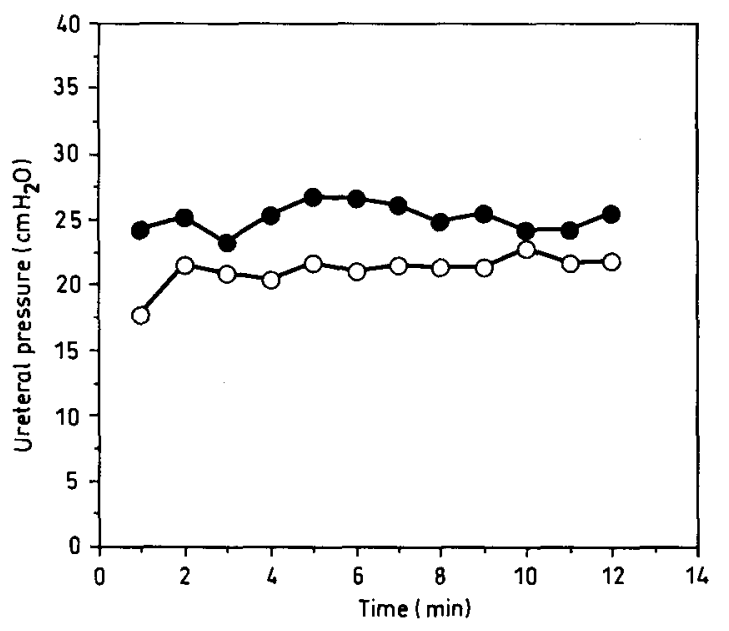

Fig. 3. Typical open bladder outlet in a myelodysplastic boy (decentralized bladder). Outlet resistance is the result of relatively fixed external sphincter activity. OAcute group; - chronic group. Perfusion rate is $0.191 \mathrm{ml} / \mathrm{min}$

totally unexpected. The volume that each child's bladder held at a pressure of $40 \mathrm{~cm} \mathrm{H}_{2} \mathrm{O}$ increased very substantially. These results indicated that a high, fixed outlet resistance could induce a change in compliance that was reversible on a decrease in outlet resistance, implying that at least in this circumstance, the poorly compliant bladder was the result of an elevated, fixed outlet resistance.

The third set of observations involved the use of a feline model of obstructive uropathy. A silastic cuff was placed around the urethra of cats so as to raise the voiding pressure from a mean normal of about $16 \mathrm{~cm} \mathrm{H}_{2} \mathrm{O}$ to $20-120 \mathrm{~cm}_{2} \mathrm{O}$. After implantation, the animals were observed for 1 year, at which time a series of experiments were done to compare the findings in long-term obstruction with those in normal controls and in a small group of cats whose urethras had been obstructed for a very short period. The upper ureters in the long-term obstructed group were cannulated and perfused at three rates, and identical studies were done in controls. The pressure required to drive urine across the ureter into the bladder was from 2 to $4 \mathrm{~cm} \mathrm{H}_{2} \mathrm{O}$ higher in the chronically obstructed animals than in normal cats (Fig. 3). In both obstructed and normal animals, however, there was a linear relationship between ureteral perfusion pressure and intravesical pressure. At any bladder pressure and volume, ureteral perfusion pressure remained higher than bladder pressure.

In another series of experiments, seven animals that had undergone acute, total obstruction were compared with normal controls on day 7. The obstructed animals were managed during the 7-day period by intermittent catheterization. In acutely obstructed and normal animals, the passive responses of the ureters to perfusion indicated that at all perfusion pressures from 5 to $50 \mathrm{~cm}$ $\mathrm{H}_{2} \mathrm{O}$, obstructed ureters allowed the transfer of a larger volume of fluid than did normal ureters. However, when the bladder and ureter were kept at the same pressure, the amount of fluid transferred by active ureteral work was greater in normal ureters than in obstructed ones. These findings have important clinical implications, the most obvious being that a wide ureter may have better perfusion characteristics than a normal ureter. In addition, the determination that a ureter can peristaltically transfer sufficient urine to avoid trouble is presently not precisely directly measurable. For instance, if the bladder compliance is abnormal and the limit of urethral resistance is $34 \mathrm{~cm} \mathrm{H}_{2} \mathrm{O}$, with the ureters thus facing a mean bladder pressure of about $20 \mathrm{~cm} \mathrm{H}_{2} \mathrm{O}$, will the ureters continue to function normally? The answer is that no one knows how to measure that variable at present. It is known that a ureter faced with a mean intravesical pressure of $40 \mathrm{~cm} \mathrm{H}_{2} \mathrm{O}$ will dilate, decompensate, exhibit vesicoureteral reflux, and ultimately adversely influence renal function, but exactly how the last event occurs remains unknown, and the variables of pressure, time, and urinary flow rate that induce ureteral and renal failure are not precisely known.

\section{Discussion}

These interrelated clinical and experimental findings are of great importance to our understanding of the relationship between lower urinary tract function and upper tract maintenance and survival. Since it seems clear that intravesical pressure is the most important single factor in terms of the maintenance of normal upper tract function, control of that variable should be the cardinal urologic effort. Control of vesical pressure along with preservation of the normal storage behavior of the bladder is possible through either a reduction in outlet resistance or an increase in bladder capacity obtained using surgery or drugs. The observation that compliance deteriorates progressively in the presence of fixed or reflexive elevated outlet resistance and that this process is in some circumstances reversible by a reduction in outlet resistance implies the development of a kind of compensatory mechanism, even by a decentralized bladder, when this organ is faced with fixed outlet resistance. The mechanism involved are presently unknown, but the degree of loss of compliance appears to be related to the magnitude of the outlet resistance and to the period over which intravesical pressure and outlet resistance interact.

If began early, intermittent catheterization may totally prevent the development of poor compliance in specific circumstances. The evidence for this effect of intermittent catheterization comes from both myelodysplastic children and patients with spinal cord injury. In the latter patients, poor compliance and, to a considerable extent, overt detrusor-sphincter dyssynergia can be prevented by the early implementation of intermittent catheterization. In the current Michigan Spinal Cord Injury Series, $96 \%$ of 118 patients are dry and maintain low-pressure bladder urinary reservoirs on intermittent catheterization protocols and one-third of the patients require no ancillary drug therapy to maintain that status. Over a mean followup of 38 months, this situation has easily been maintained.

These results contrast with efforts to prevent upper urinary tract damage or changes by constant Foley or suprapubic tube-catheter drainage, whereby a progressive 
cumulative risk to ureteral and upper tract function exists despite maintenance of a nearly empty bladder. The mechanisms underlying this kind of dismal outcome in patients treated by tubes are currently unknown but may be related to local effects on the ureter at its junction with the bladder, to progressive vesical fibrosis, or to other as yet unidentified processes.

On a basic clinical level, the physician who is confronted with patients showing ureteral dilation as diagnosed by X-ray or ultrasound study cannot by simple observation immediately determine whether an active obstructive process is involved. A cystometrogram that shows a normal filling curve helps to rule out the bladder as a source of the ureteral enlargement. Moreover, despite some excellent experimental work, it is not clear that a dilated human ureter invariably results in poor or inadequate function. For example, patients considered for undiversion because of upper urinary tract stones or disease who have long-standing ureteral dilation, which in fact prompted the diversion in the first place, cannot be considered inoperable only because the ureters are large.

Perfusion at $10 \mathrm{ml} / \mathrm{min}$ into an ileal loop or out of a urostomy stoma demonstrates, as Whitaker [3] originally intended, that the ureter has no mechanical obstruction, but that is not the same thing as either reserve power or peristaltic ability. At high flow rates, peristaltic activity is said to be less important, but our data in this regard was obtained in experimental animal studies conducted only in ureters not draining into a variably compliant vesicoelastic reservior. Thus, we do not have precise information on the relative importance to ureteral function of active contractility as opposed to the hydrostatic pressure of urine formation. However, in the aggregate, both the hydrostatic pressure of urine formation pressure and peristaltic activity fail to move urine at sustained intravesical pressures of $>40 \mathrm{~cm} \mathrm{H} \mathrm{H}_{2} \mathrm{O}$. Between values of 20 and $40 \mathrm{~cm} \mathrm{H}_{2} \mathrm{O}$ exists a kind of grey zone. Except in untreated states characterized by detrusor areflexia and by the fixed outlet resistance and constant dribbling urinary loss that are encountered in end-stage prostatism (very rarely), diabetes mellitus (also rarely), and myelodysplasia (not so rarely), bladder pressures are seldom constant but are related to volume, to residual urine, and to outlet resistance. Thus, the present study did not define either the bladder pressures that can induce ureteral deterioration over protracted periods or the outlet resistance that enables this to occur over time.

We need a better understanding of the mechanisms involved in the effects of outlet resistance on bladder storage function or compliance so as to deal with this problem without resorting to surgical procedures on the outlet that obviate continence. Also, a more precise method for the measurement of both active and passive ureteral function is needed.

\section{References}

1. Coolsaet BLRA, van Venrooij GEPM, Blok C (1982) Detrusor pressure versus wall stress in relation to ureterovesical resistance. Neurourol Urodyn 1:105-111

2. Bloom DA, Knechtel JM, McGuire EJ (1990) Urethral dilation improves bladder compliance in children with meningomyelocelis and high leak point pressures. J Urol (in press) 